\title{
Mathematical Modeling and Optimization of Industrial Problems
}

\author{
M. Montaz Ali, ${ }^{1}$ Aderemi O. Adewumi, ${ }^{2}$ Nachamada Blamah, ${ }^{2,3}$ and Olabisi Falowo ${ }^{4}$ \\ ${ }^{1}$ Centre for Systems and Engineering, Faculty of Engineering, University of the Witwatersrand, Johannesburg, South Africa \\ ${ }^{2}$ School of Mathematics, Statistics \& Computer Science, University of KwaZulu-Natal, Durban, South Africa \\ ${ }^{3}$ Department of Computer Science, University of Jos, Jos, Nigeria \\ ${ }^{4}$ Department of Electrical and Electronic Engineering, University of Cape Town, Cape Town, South Africa
}

Correspondence should be addressed to M. Montaz Ali; montaz.ali@wits.ac.za

Received 11 December 2014; Accepted 11 December 2014

Copyright (c) 2015 M. Montaz Ali et al. This is an open access article distributed under the Creative Commons Attribution License, which permits unrestricted use, distribution, and reproduction in any medium, provided the original work is properly cited.

Optimization plays an ever-increasing role in mathematics, economics, engineering, health sciences, management, life sciences, and almost all other fields of study. Many optimization problems exist in the real world including space planning, networking, logistic management, financial planning, and risk management. These problems are NPhard in nature; hence, finding an optimal solution is often difficult, demanding highly efficient techniques. Many exact and heuristic algorithms have been proposed out of these challenging and practically relevant optimization problems including those that draw inspiration from nature.

The aim of this special issue is to showcase how heuristics, operations research, computational intelligence, and other optimization techniques have been successfully applied to solve real-world industrial optimization problems. Researchers were invited to contribute original research and review articles that explore, model, and solve real life optimization problems in various fields. The special issue attracted many high quality papers of which few were selected based on set quality criteria after rigorous peerreview, revision, and editorial processes. The published high quality papers cover various applications of optimization to varying problems especially in the industries including engineering, inventory, logistics, and marketing, scheduling, resource, and transportation planning, computing and ICT, water and gas resource management, and data mining. Most papers dwell on modeling of real-world industrial problems, innovative optimization technique for solving real-world problems including heuristics, finite methods, operation research techniques, intelligent algorithms, and agent-based methods. Many of the papers propose innovative techniques for solving specified problem while others present improved methods compared with existing ones. Here, we present a summary of the published papers categorized according to the problem domain addressed.

Engineering. Papers in the engineering domain cover interests in electrical, mechanical, and building engineering. L. Huo et al. present an optimal design methodology for tuned liquid column dampers (TLCDs) based on the $H_{\infty}$ control theory using the Dalian Xinghai Financial Business Building as case study in their paper titled "TLCD Parametric Optimization for the Vibration Control of Building Structures Based on Linear Matrix Inequality."

Y.-C. Park et al. present a "Lightweight Design of an Outer Tie Rod for an Electrical Vehicle" which was achieved through efficient material selection using finite element analysis and metamodel-based optimization technique in order to minimize the weight while satisfying critical design requirements. Furthermore, the paper "Hierarchical AgentBased Integrated Modelling Approach for Microgrids with Adoption of EVs and HRES" by P. Han et al. addresses the challenge to microgrid, brought about by the recent adoption of electric vehicles and hybrid renewable energy systems, by proposing a hierarchical agent-based method which is useful for energy management, electricity consumption prediction, the EV scheduling control, and HRES deployment optimization. Finally in electrical, "Predictive Models of Current, 
Voltage, and Power Losses on Electric Transmission Lines" O. M. Bamigbola et al. formulated mathematical physics expressions that depict the evolution of current and voltage on a typical transmission line and derived models to predict available current and voltage at any point on the transmission line.

In the paper "Partitioned Quasi-Newton Approximation for Direct Collocation Methods and Its Application to the Fuel-Optimal Control of a Diesel Engine," J. Asprion et al. presented an efficient technique to resolve memory limitations, preserve the correct sparsity pattern, and generate more accurate curvature information as it applies to engineering problems with special focus on fuel-optimal and emissionconstrained control of a turbocharged diesel engine. "Finite Queueing Modeling and Optimization: A Selected Review" by F. R. B. Cruz and T. van Woensel is a comprehensive review of queueing modeling issues and related performance evaluation and optimization approaches for joint manufacturing and product engineering.

D. Xiao et al. in their paper "Quality Prediction and Control of Reducing Pipe Based on EOS-ELM-RPLS Mathematics Modeling Method" present an algorithm to predict the quality of reducing pipe during the inspection of inhomogeneous transverse and longitudinal wall thicknesses during the production of seamless steel. In "Three-Dimensional Temperature Distribution Produced by a Moving Laser Beam" R. Uyhan used a simplified theoretical model to study the threedimensional unsteady temperature field produced by moving laser beam in an axisymmetric laser beam.

The Computing and ICT. The Computing and ICT groups of papers dwell on computer graphics, multiprocessor computing, wireless communication, electronic fraud prevention, and robotics. X. Wang and C. Hou in the paper "Improved Crosstalk Reduction on Multiview 3D Display by Using BILS Algorithm" present a system-introduced crosstalk measurement and derive an improved crosstalk reduction method to address the problem of crosstalk that degrades the quality of the 3D image. Their improved method seeks an optimal solution to this box-constrained integer least squares (BILS) problem that substantially decreases the Euclidean distance between a solution and its target. The paper "An Efficient Biobjective Heuristic for Scheduling Workflows on Heterogeneous DVS-Enabled Processors" by P. Zhou and W. Zheng addressed the problem of energy consumption, expressed in terms of execution time of application, in multiprocessing computing. The authors proposed a heuristic that finds a schedule for a workflow, on a bounded number of heterogeneous (dynamic voltage scaling) DVS-enabled processors, so as to minimize both makespan (overall execution time of the application) and energy consumption.

Furthermore, C. Han et al. in their paper, "Modeling and Model Predictive Power and Rate Control of Wireless Communication Networks," present a novel power and rate control system model for wireless communication networks and proposed a delay-dependent model predictive power and rate control method. The derived optimization model is solved using linear matrix inequality (LMI) technique. Finally, A. A. Akinyelu and A. O. Adewumi in "Classification of Phishing Email Using Random Forest Machine Learning Technique" propose an efficient random forest technique for phishing email detection.

The paper "Robot Calibration for Cooperative Process under Typical Installation" by Y. Gan et al. introduces simple but efficient operation procedure and calibration condition to solve the base frame calibration problem for cooperative robots which was validated on two practical industrial robots. In "Track-to-Track Association Based on Structural Similarity in the Presence of Sensor Biases," H. Zhu and S. Han address the problem of track-to-track association in the presence of sensor biases by introducing the structural feature for each local track, which describes the spatial relationship with its neighbouring targets.

Inventory, Logistics, and Marketing. There are five papers in the inventory control, logistics, and marketing applications. The paper titled "Intelligent Inventory Control via Ruminative Reinforcement Learning” by T. Katanyukul and E. K. P. Chong proposed a new ruminative reinforcement learning (RRL) method for inventory management with promising results. Similarly, the challenge of trim loss and inventory in paper industry was addressed by F.-K. Wang and F.-T. Liu in their paper. In a bid to optimize order allocation and cutting trim loss, the authors present a new decision model based on the adjustment of scheduling and limitation of inventory quantity to differentiate trim loss and inventory distribution data. Furthermore, "An Inventory Model under Trapezoidal Type Demand, Weibull-Distributed Deterioration, and Partial Backlogging" by L. Zhao proposed an inventory model and policy for Weibull-distributed deterioration items with trapezoidal type demand rate, in which shortages are allowed and partial backlogging depends on the waiting time for the next replenishment.

D. Kim in the paper "A Dynamic Model of the Tragedy of the Commons in Marketing-Intensive Industries" provides the analysis of the processes of a dynamic model introduced to prevent business ecosystem from being plunged into Tragedy of the Commons (ToC) thereby enhancing sustainable growth and competitiveness of the business ecosystem.

Finally, in "Metaheuristic Algorithm for Solving Biobjective Possibility Planning Model of Location-Allocation in Disaster Relief Logistics" by F. Barzinpour et al., a multiobjective model for periodic location and allocation of distribution centres to damaged areas during natural disaster in order to distribute relief commodities is presented and solved using GA.

Water and Gas Resource Management. There are also five papers dealing with water, oil, and gas resource management. Y. Wang et al. in their paper "An Analytical Solution by HAM for Nonlinear Simulation of Deepwater SCR Installation" seek to optimize the time taken during installation path calculation by numerical simulation software in steel catenary riser (SCR) by establishing a mechanical model that makes use of homotopy analysis method to simplify its analytical solution while dimensional analysis was considered in making initial guess solution. X.-H. Tan et al. establish a new mathematical model of gas-water two-phase 
flow to "Determine the Inflow Performance Relationship of Water Producing Gas Well Using Multiobjective Optimization Method" thus overcoming the limitation of well testing on site. Also, in "Numerical Investigation of Gas Mixture Length of Nitrogen Replacement in Large-Diameter Natural Gas Pipeline without Isolator" by H. Zhu and Q. Han, a computational fluid dynamic model coupled to a speciestransportation model was used to investigate the gas mixture length of nitrogen replacement in large-diameter pipeline without isolator.

In "Modeling and Optimization of Beam Pumping System Based on Intelligent Computing for Energy Saving," X. $\mathrm{Gu}$ et al. present a novel intelligent technique for optimizing beam pumping system for petroleum enterprises in China in order to save energy. In "Mathematical Model of Pipeline Abandonment and Recovery in Deepwater," X.-G. Zeng et al. deal with the challenge of pipeline abandonment and recovery in offshore oil and gas engineering by introducing a third-order differential equation which is solved using a novel technique.

Scheduling, Resource, and Transportation Planning. A few papers consider scheduling, resources, and transport planning within different domain area. The paper "A Scheduling Problem in the Baking Industry" by F. A. M. da Silva et al. presents greedy and genetic algorithm (GA) to handle a real-world scheduling problem in the baking industry arising from increasing production rates, growing demand, and resource constraints. The main objective is to optimize both delivery time and profit maximization. The paper "Multiobjective Fuzzy Mixed Assembly Line Sequencing Optimization Model" by F. Tahriri et al. addresses the research gap in the assembly line sequencing optimization model for mixedmodel production lines by presenting an integrated model of job shop and assembly production lines for factories with modular layouts that seeks to minimize the makespan, setup time, and cost simultaneously in the mixed-model assembly lines. GA was employed to solve the derived model.

S. M. Akandwanaho et al. in "Solving Dynamic Traveling Salesman Problem Using Dynamic Gaussian Process Regression" present a hybridization of dynamic Gaussian process regression with nearest neighbor and iterated local search to the track dynamic optima for the dynamic traveling salesman problem. In "Airline Overbooking Problem with Uncertain No-Shows," C. Zhang et al. present an idea that seeks to minimize the effect of no-shows and any other uncertain human behaviour or unexpected events in an airline overbooking problem of a new single-leg flight with discount fare. "Centralized Resource Allocation for Connecting Radial and Nonradial Models" by A. Mirsalehy et al. examines an alternative approach to the centralized resource allocation model that indicates that all units are under the control of an entity of the centralized decision maker.

"Flow Merging and Hub Route Optimization in Collaborative Transportation" by K. Weng and Z. Xu studies the optimal hub routing problem of merged tasks in collaborative transportation being applicable in logistics, postal services, airline, and other transportation systems and presents two heuristics to solve the derived mixed integer programming model. S. Wen et al. in "Weighted Multimodel Predictive Function Control for Automatic Train Operation System" design a speed controller for train operation based on the input and output data of the train in order to obtain a predictive model about the system based on weighted indicators.

In "New Product Development in an Emerging Economy: Analysing the Role of Supplier Involvement Practices by Using Bayesian Markov Chain Monte Carlo Technique," $\mathrm{K}$. Kanapathy et al. use structural equation modelling and Bayesian Markov Chain Monte Carlo algorithm in a case of 146 companies in China to determine whether the positive relationship found between supplier involvement practices and new product development performances in developed economies also holds in emerging economies.

Data Mining. L. Si et al. present "A Novel Classification Approach through Integration of Rough Sets and BackPropagation Neural Network" which essentially hybridizes the underlying techniques for application in data mining. P. Santos et al. in their paper "Modelling Laser Milling of Microcavities for the Manufacturing of DES with Ensembles" study the laser-milling process of microcavities in the manufacture of drug-eluting stents (DES). A set of designed experiments using different regression methods (ensemble, support vector, $\mathrm{ANN}$, and linear and nearest neighbour) were conducted with ensemble regression as the most efficient technique.

In conclusion, this special issue has contributed to the field of optimization and modelling of real-world problem as well as development of innovative optimization techniques for handling such problem. We hope that ideas generated in this issue will provoke further research and thoughts in the field of mathematical modelling, optimization, and optimization techniques.

\section{Acknowledgment}

We would like to express our profound gratitude to all the reviewers whose professional expertise contributed immensely to the outcome of this special issue.

M. Montaz Ali Aderemi O. Adewumi Nachamada Blamah Olabisi Falowo 


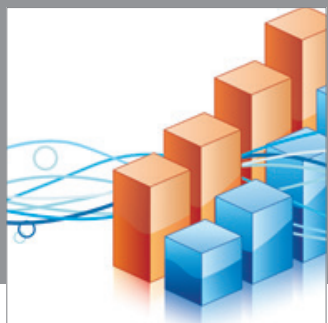

Advances in

Operations Research

mansans

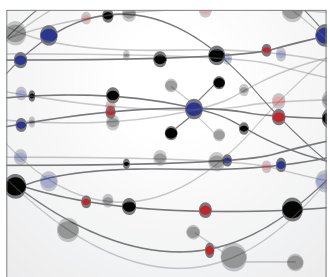

The Scientific World Journal
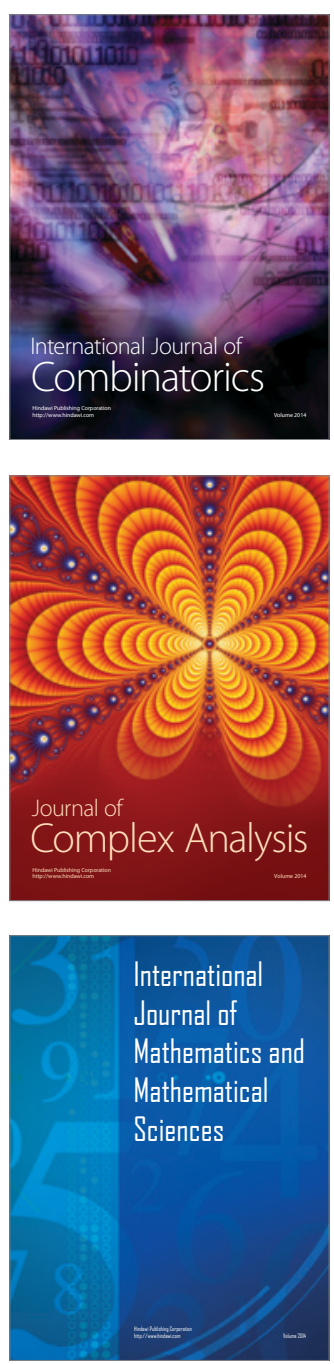
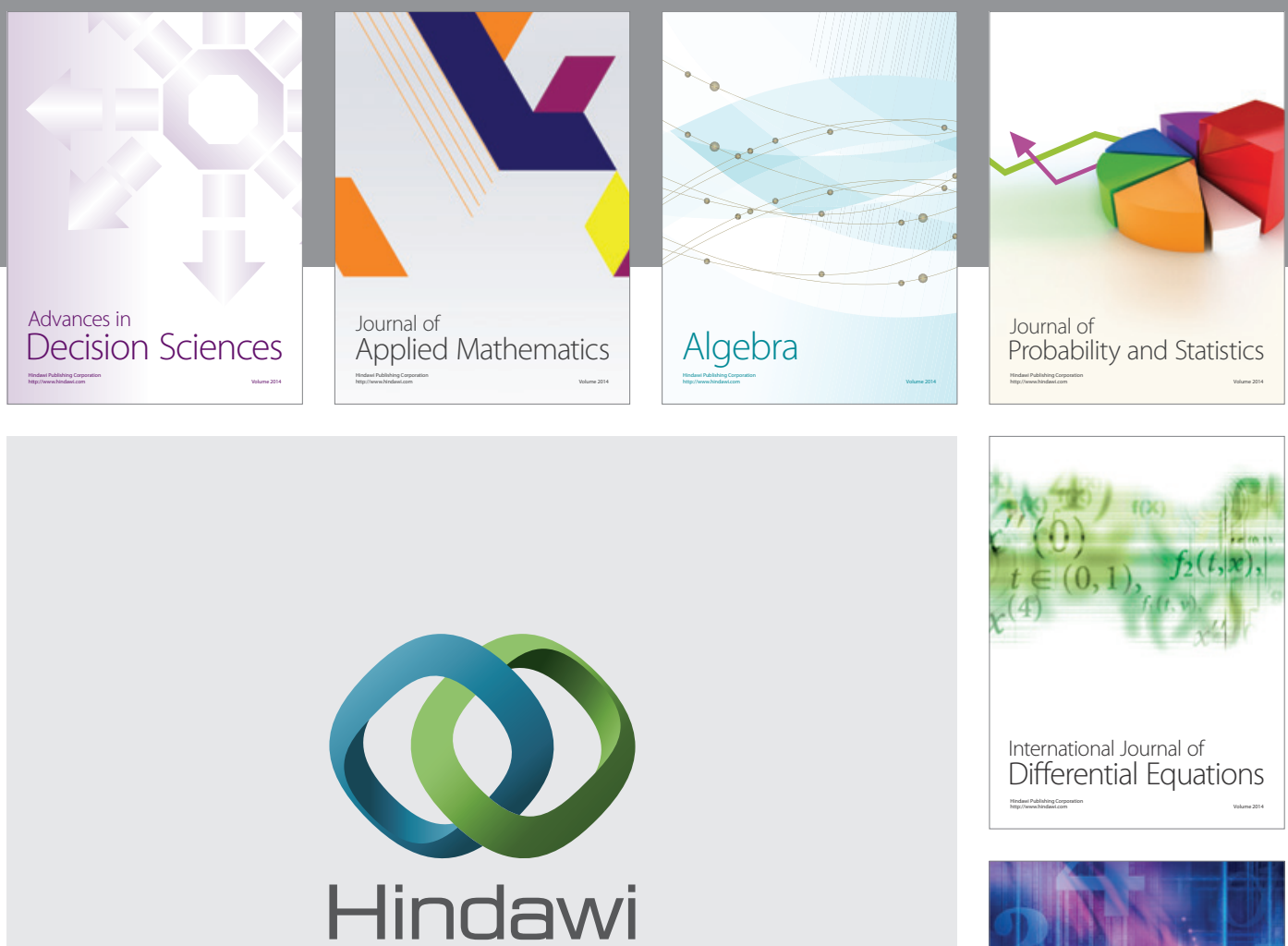

Submit your manuscripts at http://www.hindawi.com
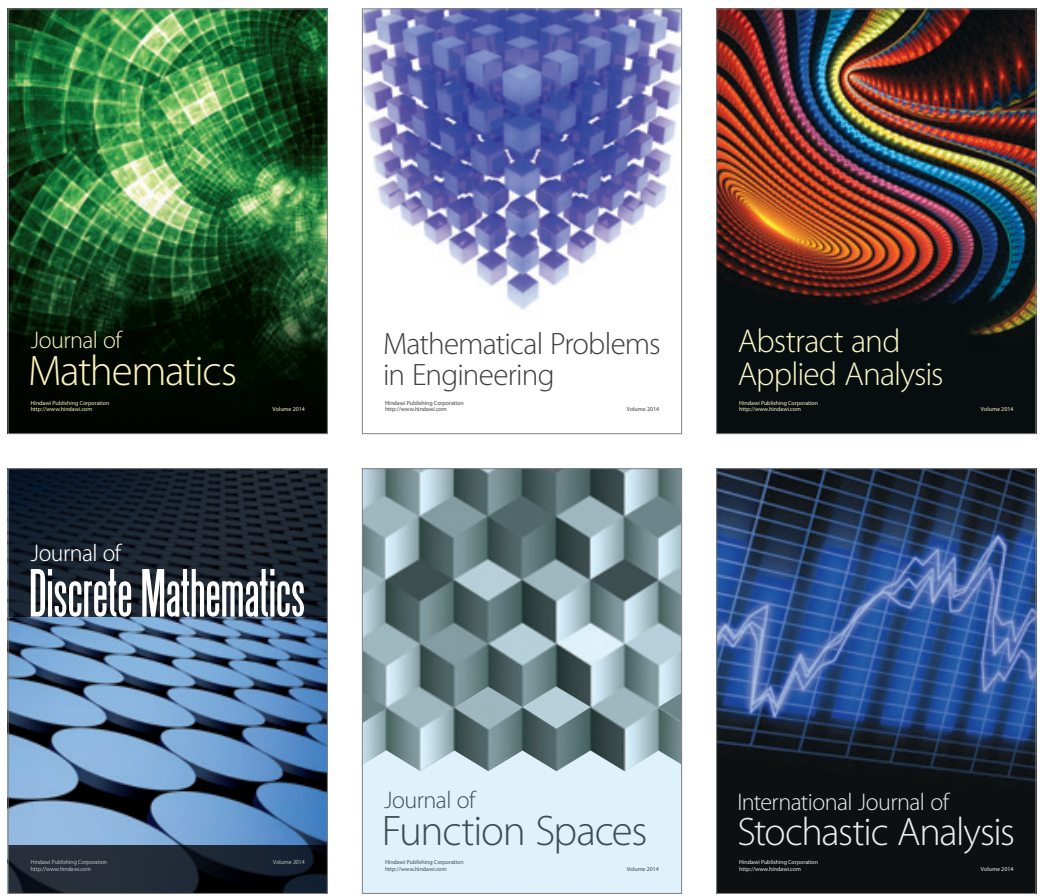

Journal of

Function Spaces

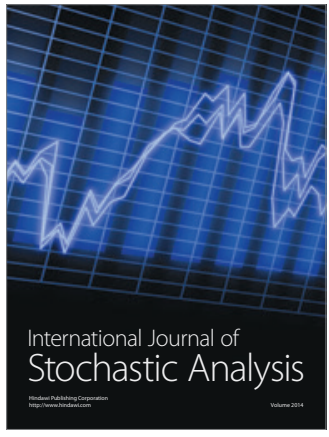

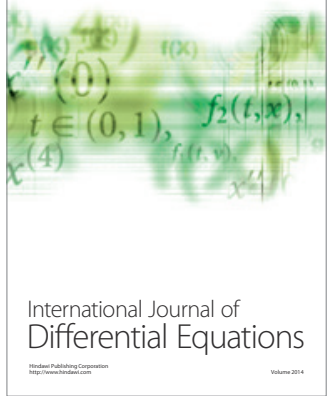
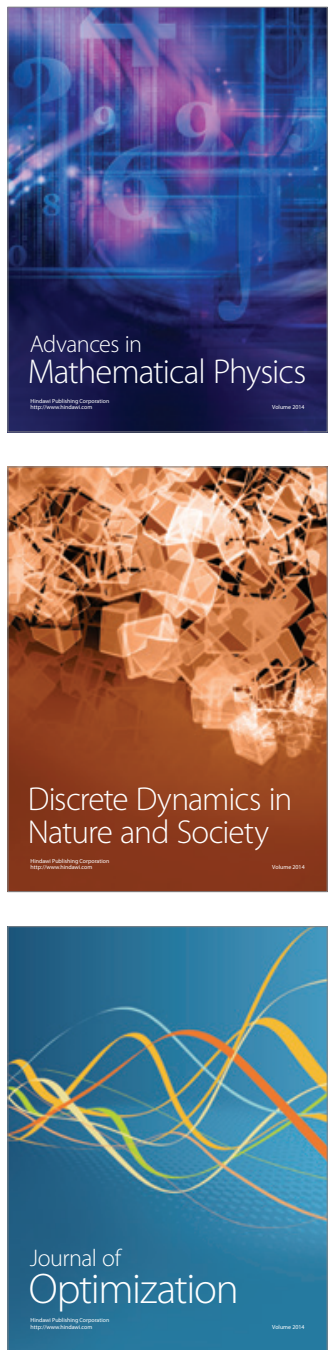